\title{
Validación de apariencia, contenido y consistencia interna de la Escala de Apreciación de Agencia de Autocuidado (ASA) para Costa Rica, segunda versión en español para población conocida sana ${ }^{1}$
}

Institución: Universidad de Costa Rica

Leiva Díaz Viriam² Cubillo Vargas Kenneth ${ }^{3}$ Porras Gutiérrez Yuliany ${ }^{4}$ Ramírez Villegas Tatiana ${ }^{5}$ Sirias Wong Isabel ${ }^{6}$

\section{COMO CITAR}

Leiva, V., Cubillo, K., Porras, Y., Ramírez, T. y Sirias, I (2016). Validación de apariencia, contenido y consistencia interna de la Escala de Apreciación de Agencia de Autocuidado (ASA) para Costa Rica, segunda versión en español para población conocida sana. Rev. Enfermería Actual de Costa Rica, 30, 1-20. DOI: http://dx.doi.org/10.15517/revenf.v0i30.22110

\section{RESUMEN}

Introducción. Este artículo presenta los resultados obtenidos del proceso de validación de apariencia, contenido y consistencia interna al que fue sometida la segunda versión en español de la escala ASA con población costarricense conocida sana.

Metodología. Se trata de un estudio descriptivo, transversal, de enfoque cuantitativo, diseño no experimental y psicométrico, realizado con una muestra de 105 personas en etapa de adultez temprana, intermedia y tardía. Resultado. La investigación determinó que el estudio factorial (método ACP) con rotación Varimax sugirió el análisis con cinco ejes factoriales que explican el 60,03\% de la varianza total en Costa Rica. Del resultado de los dominios anteriores, se destaca que en la mayoría de ellos predomina la categoría relacionada al requisito universal de prevención de peligros contra la vida, el funcionamiento y bienestar humano.

Conclusión. En Costa Rica, la enfermería puede contar con un instrumento válido y confiable para medir la capacidad de agencia de autocuidado en las personas conocidas sanas que se encuentran en la etapa de la adultez.

Palabras Clave. Consistencia-interna, validación-apariencia, validación-contenido, personas-sanas

\footnotetext{
${ }^{1}$ Fecha de recepción: 20 de febrero 2015

Fecha de aceptación: 9 de mayo 2015

${ }^{2}$ Enfermera. Profesora Catedrática de la Universidad de Costa Rica. Escuela de Enfermería. Licenciada en Psicología, Magíster en Psicopedagogía. Correo electrónico: viriaml@gmail.com

${ }^{3}$ Licenciado en Enfermería. Profesor Universidad de Costa Rica. Escuela de Enfermería. Correo electrónico: kenncub@hotmail.com

${ }^{4}$ Licenciada en Enfermería. Correo electrónico: yulianyp@gmail.com

${ }^{5}$ Licenciada en Enfermería. Hospital Cima. Correo electrónico: tatyanra@yahoo.es

${ }^{6}$ Licenciada en Enfermería. Correo electrónico: isw87@hotmail.com
} 


\title{
Validation appearance, content and internal consistency of the scale Appraisal of Self-care Agency (ASA) to Costa Rica, for second Spanish version known population healthy ${ }^{1}$
}

Institution: University of Costa Rica

Leiva Díaz Viriam² Cubillo Vargas Kenneth ${ }^{3}$ Porras Gutiérrez Yuliany ${ }^{4}$ Ramírez Villegas Tatiana ${ }^{5}$

Sirias Wong Isabel ${ }^{6}$

\section{CITED:}

Leiva, V., Cubillo, K., Porras, Y., Ramírez, T. y Sirias, I (2016). Validation appearance, content and internal consistency of the scale Appraisal of Self-care Agency (ASA) to Costa Rica, for second Spanish version known population healthy. Rev. Enfermería Actual de Costa Rica, 30, 1-20. DOI: http://dx.doi.org/10.15517/revenf.v0i30.22110

\begin{abstract}
Introduction. This article presents the results of the validation process of appearance, content and internal consistency that was submitted the second version in Spanish of the ASA scale known Costa Rican population healthy.

Methodology. This is a transversal, descriptive study of quantitative approach, not experimental and psychometric design, conducted with a sample of 105 people on stage early, middle and late adulthood.

Result. The investigation determined that the factorial study (ACP method) with Varimax rotation analysis suggested five factorial axes that explain $60.03 \%$ of the total variance in Costa Rica. The outcome of the above domains highlighted that in most of them the universal requirement category related to prevention of hazards to life, the functioning and human well-being prevails.

Conclusion. In Costa Rica, the nurse may have a valid and reliable to measure the ability of self-care agency known in healthy people who are at the stage of adulthood instrument
\end{abstract}

Key Words. Appearance-validation, content-validation, internal-consistency, persons-know-healthy.

\footnotetext{
${ }^{1}$ Date of acceptance: February 20, 2015

Date of receipt: May 9, 2015

${ }^{2}$ Enfermera. Profesora Catedrática de la Universidad de Costa Rica. Escuela de Enfermería. Licenciada en Psicología, Magíster en Psicopedagogía. Correo electrónico: viriaml@gmail.com

${ }^{3}$ Licenciado en Enfermería. Profesor Universidad de Costa Rica. Escuela de Enfermería. Correo electrónico: kenncub@hotmail.com

${ }^{4}$ Licenciada en Enfermería. Correo electrónico: yulianyp@gmail.com

${ }^{5}$ Licenciada en Enfermería. Hospital Cima. Correo electrónico: tatyanra@yahoo.es

${ }^{6}$ Licenciada en Enfermería. Correo electrónico: isw87@hotmail.com
} 


\section{Revista Electrónica Enfermeria Actual en costa Rica}

\section{INTRODUCCIÓN}

El acto de cuidarse es natural e inherente al ser humano y es tan fundamental como lo es el sobrevivir, por tanto, al asumir el autocuidado se promociona la vida y el bienestar, se previene la enfermedad y, a su vez, la recuperación de ella, motivo por el que toda acción que la persona realiza conlleva implícitamente el autocuidado (Fernández y Manrique-Abril. 2010).

La Teoría de Autocuidado, desarrollada por Dorothea Orem en 1983, acuña el término de la agencia de autocuidado, definida como "la capacidad de participar en el autocuidado" (p.112), término que se constituye en el fundamento de instrumentos a nivel internacional como la escala de Ejercicio de Agencia de Autocuidado (ESCA), el Cuestionario de la Estructura Cognitiva relacionado con salud y autocuidado y el cuestionario de percepción de la agencia de Autocuidado (PSCA) (Achury, Sepúlveda y Rodríguez, 2008); sin embargo, uno de los instrumentos que recoge los principales elementos de la capacidad de autocuidado por evaluar en las personas, de forma más clara y concisa, es el desarrollado en Holanda por Evers, Isenberg, Philipsen, Senten y Brouns en 1983 (Rodríguez, Díaz, 2011) denominado Escala de Apreciación de Agencia de Autocuidado (ASA, por sus siglas en inglés, Appraisal of the Selfcare Agency)

En relación con lo anterior, para desarrollar la capacidad de autocuidado, los individuos deben contar con tres elementos importantes: capacidades fundamentales y disposición de autocuidado, las capacidades para operaciones de autocuidado y los componentes de poder (Rivera, 2006; Rivera y Díaz, 2007); este último elemento fue el que los autores de la escala $A S A$ eligieron para sustentar la escala que contempla diez componentes: máxima atención o vigilancia, control de la energía física, control del movimiento del cuerpo, habilidad para razonar, motivación para la acción, habilidades para tomar decisiones, conocimiento, repertorio de habilidades, habilidades para ordenar las acciones de autocuidado y habilidades para integrar (Evers, 1998).

Dicha escala fue traducida al español en 1998 por Gallegos y ha sido utilizada en varios países como Guatemala (Porras, 2002), Nicaragua, (Ortiz, 2002), Honduras (Monge 2004) y Colombia (Rivera, 2006) y en países de Europa, Asia Oriental y Latinoamérica, tales como México, Hong Kong, Suiza, Noruega, Dinamarca, España, Turquía, Estados Unidos, entre otros (Manrique-Abril, Fernández y Velandia 2009), por lo que se ha validado el constructo, lo cual "demuestra el poder del instrumento para discriminar grupos con diferencias conocidas en su nivel de dependencia" (Velandia y Rivera, 2009: p.40).

En el caso del instrumento traducido al español en México, su formato original tenía una escala de Likert de 5 alternativas de respuesta (totalmente en desacuerdo, en desacuerdo, indeciso, de acuerdo y totalmente de acuerdo); sin embargo, lo cual cambió en el contexto colombiano en donde pasó a tener 4 alternativas: nunca, casi nunca, casi siempre y siempre y los ítems 4, 6, 13 y 16 fueron reescritos de manera más simple y gramaticalmente correctos (Velandia y Rivera, 2009), siempre conservando el rango de 24 a 96 puntos. (Espitia, 2011).

A la escala en Colombia se le aplicó un análisis factorial a la población con patología crónica, el cual demostró que poseía propiedades psicométricas válidas (Manrique-Abril, Fernández y Velandia, 2009), así como su confiabilidad, de lo que resultaron 24 ítems que medían un mismo concepto, característica que le confirió consistencia interna (Velandia y Rivera, 2009). 
Para desarrollar la validación de un instrumento se debe seguir diversos pasos como desarrollar la validez de apariencia, la validez de contenido y la consistencia interna de la Escala de ASA en su segunda versión en español en Costa Rica dirigido a personas adultas sanas, los cuales fueron el objetivo de esta investigación.

\section{MATERIALES Y MÉTODOS}

Este estudio es de tipo descriptivo y transversal, con un enfoque cuantitativo y un diseño no experimental (Hernández, Fernández y Baptista, 2006). A su vez fue psicométrico (Campos y Obviedo, 2008), puesto que persiguió adaptarse al contexto costarricense (Kerlinger y Lee, 2002).

En las siguientes viñetas se ofrece un resumen de los pasos desarrollados en la investigación.

- Para validar la apariencia, se requirió del criterio de cinco expertos (Contreras, Flórez y Herrera, 2008) en las áreas enfermería, estadística, filología y psicología (específicamente en psicometría) y cinco personas ajenas a la población meta que cumplieran con los criterios de inclusión. Se analizó los criterios aportados para determinar su pertinencia (Sánchez y Echeverri, 2004).

- En la validez de contenido, inicialmente se determinó la viabilidad mediante la prueba de Kaiser-MeyerOlkin (KMO) y la prueba de esfericidad de Barlett, (Lamprea y Gómez, 2007), luego se le aplicó el instrumento a la muestra, una matriz de correlación y el análisis factorial utilizando la técnica de extracción de componentes principales y la de rotación Varimax.

- En el proceso de consistencia interna se procedió a estimar la confiabilidad de la escala por medio del índice estadístico de Alfa de Cronbach (George y Mallery, 1995).

La unidad de análisis fue la persona adulta, para lo que se consideró los periodos de desarrollo descritos por Papalia (2005) como etapa de la adultez temprana (20-40 años), intermedia (40-65 años) y tardía (65 años en adelante): las primeras dos etapas son abarcadas por todos los funcionarios del INS Salud, mientras que la población adulta tardía del Programa Integral de la Persona Adulta Mayor (PIAM) de la Universidad de Costa Rica, matriculadas en el primer semestre del año 2011.

La muestra de tipo no probabilístico por cuotas (Hernández, Fernández y Baptista, 2006) estuvo constituida por 211 personas adultas en total, distribuidas en cada etapa equitativamente de las cuales 105 personas fueron conocidas sanas y 106 con algún tipo de enfermedad crónica; sin embargo, el presente artículo hará referencia solo a la validación de la escala con la muestra de personas conocidas sanas, razón por la que se comprenderá salud según lo manifestado por la OMS/OPS en el 2007, entidad que la define como

un fenómeno con implicaciones sociales, económicas y políticas y, sobretodo, un derecho fundamental, y las desigualdades, la pobreza, la explotación, la violencia y la injusticia están entre las causas más importantes de los problemas de salud que afectan a la gente pobre y marginada (2007, s.p.). 
Partiendo de lo mencionado, la muestra de personas sanas incluye personas que refirieron no tener ninguna enfermedad como también personas con problemas o enfermedades agudas o transitorias.

Por otra parte, el tamaño muestral se determinó considerando que

no existe un criterio o norma definitiva sobre el número de sujetos necesario. En principio son preferibles muestras grandes, porque el error típico de los coeficientes de correlación será menor y de esta manera disminuye también la probabilidad de que surjan factores casuales que no aparecerán en análisis sucesivos con otras muestras (Morales, 2011, p.8).

A pesar de lo anterior, Norman y Streiner (1996), citados por Sánchez y Echeverri (2004) mencionan que "para efectuar este tipo de análisis se requieren, por lo menos, cinco pacientes por cada item que tenga el instrumento, pero no menos de 100 pacientes en total".

La recolección de los datos se llevó a cabo durante el periodo de marzo a agosto del 2011, por medio de un cuestionario autoadministrado individual que constó de cuatro partes: la primera fue la guía para validación de jueces expertos (aplicada únicamente a los 5 expertos seleccionados); la segunda, la de datos sociodemográficos por medio de preguntas abiertas y cerradas; la tercera, la escala de Capacidad de Agencia de Autocuidado y una última parte destinada a recolectar la percepción personal acerca del formato, redacción y capacidad de comprensión de la e scala por parte de los participantes.

Las variables consideradas responden a tres dimensiones: datos socio-demográficos (nacionalidad, sexo, edad, ocupación, lugar de residencia, grado de escolaridad, estado civil, ingresos económicos); estado de salud (incluye enfermedad, tratamiento farmacológico, tratamiento no farmacológico, seguro de salud) y por último se consideró las variables de alimentación, actividad y descanso, soledad e interacción social, prevención de peligros contra la vida, el funcionamiento y bienestar personal y promoción del funcionamiento y desarrollo humano.

Entre los criterios de inclusión están: hombre y mujer en la etapa de adultez, personas conocidas sanas, de indistinta nacionalidad, estatus socioeconómico, estado civil y nivel educativo, residente en Costa Rica.

\section{Consideraciones éticas}

Decisión voluntaria de ser sujetos participantes por medio del consentimiento informado. Además se consideró la autonomía, beneficencia y no maleficencia, asesoramiento y la presentación y divulgación de los resultados (Leiva, 2009).

Para analizar la información la experta en estadística de la Universidad de Costa Rica brindó asesoría; los datos fueron procesados con el paquete estadístico CSPro versión 4.1 y el programa SPSS versión 18.0, los cuales fueron exportados luego al paquete Excel 2010. 


\section{Revista Electrónica Enfermeria Actual en costa Rica}

\section{RESULTADOS}

La presentación de los resultados se realizará según las variables de estudio y se iniciará con los aspectos sociodemográficos y de cuidado.

\section{Aspectos sociodemográficos y de autocuidado}

En cuanto a la nacionalidad, 103 personas eran costarricenses, una nicaragüense y una colombiana, con un $41,9 \%$ de hombres y un $58,1 \%$ de mujeres; según su edad, un 33,3 \% (ambos sexos) se ubica en la adultez temprana, un $34,3 \%$ en la intermedia y un $32,4 \%$ en la tardía. Respecto del estado civil, predominaron las personas casadas (un 47,6\%) seguidos de las solteras (un 22,9\%) y divorciadas $(10,5 \%$ ).

En relación con la escolaridad, el grado universitario fue el que tuvo mayor representación con un 43,8\% de universidad completa y un $27,6 \%$ incompleta, seguido de la secundaria completa con un $8,6 \%$, En lo que respecta a la ocupación principal prevalecieron las ocupaciones de nivel profesional seguido del nivel técnico, en tercer lugar, las personas pensionadas y un $12,4 \%$ que no respondió a que se dedicaba. En cuanto al salario o ingreso mensual de las personas un 34,3\% afirmó recibir de $\mathbb{C} 500000$ a $\mathbb{C} 1000$ 000; un 25,7\% recibe $\mathbb{C} 1000000$ o más; un $23,8 \%$ de $\mathbb{C} 200000$ a $\mathbb{C} 500000$ y un $9,5 \%$, menos de $\mathbb{C} 200000$.

El lugar de residencia fue principalmente en la provincia de San José con un 70,5\% seguido de Heredia con un $19,4 \%$ y en tercer lugar la provincia de Cartago con un 5,8\%.

Por otro lado, un $99 \%$ afirma que cuenta con seguro de salud principalmente de la Caja Costarricense de Seguro Social, un $87,6 \%$ asiste a centros de salud, público o privado, mientras que el $12,4 \%$ restante refiere que no asiste, por las siguientes razones: ser atleta $(4,8 \%)$, porque acude hasta el momento su salud ha sido buena y no ha necesitado $(4,8 \%)$, porque prefiere acudir a servicios privados o medico de empresa $(2 \%)$ y por la mala calidad de atención en los servicios de salud (1\%).

A continuación se ofrecerá los resultados del proceso de validación de la Escala de ASA, la cual inicia con la validación de la apariencia.

\section{- Validación de apariencia}

Se realizó modificaciones estructurales de la escala ASA, cuyos ítems más difíciles se detalla continuación:

\section{Ítem 1. "A medida que cambian las circunstancias yo voy haciendo ajustes para mantener mi salud".}

En cuanto a los criterios de claridad y suficiencia, la palabra "circunstancias" debía cambiar. Se analizó su conceptualización según el Diccionario de la Real Academia Española (2011) que la define como "dicho de una cosa que de algún modo está influida por una situación ocasional", por tanto, el término se ajusta a lo que el ítem pregunta; a su vez coincide con lo planteado por Orem (1983), al afirmar que el ser humano, por naturaleza, tiene necesidades comunes para crear y mantener condiciones de vida que sostengan los procesos vitales, así como el mantenimiento y promoción de su integridad funcional. 
Dado lo anterior y partiendo de que el desarrollo humano en todas sus etapas requiere de mantener condiciones que promuevan los procesos necesarios para el avance en cada etapa del ciclo vital y de que el autocuidado está dirigido a alcanzar objetivos en distintas circunstancias, mediante acciones consecuentes, se considera un sistema de acción o un proceso dinámico que varía según las condiciones o circunstancias diarias (Orem,1983), por lo que este ítem se mantuvo redactado de la misma manera.

\section{Ítem 2. "Reviso si las formas que practico habitualmente para mantenerme con salud son buenas".}

Una de las personas con las mismas características de la población meta, así como cuatro de los expertos, comentaron que la palabra "formas" no contaba con criterios de claridad adecuados, por lo que se decidió cambiar esta palabra por la de actividades, teniendo en cuenta la recomendación de la filóloga y los fundamentos conceptuales que Orem (1983) realiza del autocuidado, definido como "la práctica de actividades que las personas inician y hacen por sí mismas para el mantenimiento de la vida, la salud y el bienestar" (p.55). Partiendo de tal premisa, Orem considera esta acción como un conjunto de actividades que el individuo realiza para tener un estado adecuado de salud y bienestar, razón por la que se modificó el ítem 2, el cual quedó redactado de la siguiente manera: reviso si las actividades que practico habitualmente para mantenerme con salud son buenas.

\section{Ítem 7. "Yo puedo buscar mejores formas para cuidar mi salud que las que tengo ahora".}

Tanto la experta en filología, estadística y una enfermera, así como una de las personas de la muestra coincidieron en que la palabra formas era ambigua, motivo por el que se decidió modificarla de la siguiente manera: "yo puedo buscar mejores acciones para cuidar mi salud que las que tengo ahora," lo cual no modifica su fundamentación teórica, ya que como Orem (1983) menciona, "el autocuidado tiene su razón de ser. Es la acción que tiene un modelo y un desarrollo y, cuando se hace eficazmente, contribuye de forma especifica a la integridad estructural, funcionamiento y desarrollo humano" (p.55); es decir, para Orem el autocuidado es una acción. Con esta adaptación del ítem se establece una redacción más clara y comprensible para las personas, se elimina la ambigüedad existente, sin perder lo que se pretende medir, en este caso si la persona realiza más acciones para cuidar la salud de las que ya practica.

Ítem 10. "Cuando hay situaciones que me afectan, yo las manejo de manera que pueda mantener mi forma de ser".

La expresión "mi forma de ser" no contaba con criterios de claridad suficientes, pues es un término subjetivo, según las expertas en enfermería. Sin embargo, al analizarlo a la luz de la teoría de autocuidado, Orem (1983) menciona los ocho requisitos universales de autocuidado en lo que respecta a la promoción de la normalidad, cuyas acciones para lograrla se enfocan en la importancia y necesidad de conservar la integridad personal o forma de ser de cada individuo ante cualquier situación que le permita estar en bienestar, mediante acciones como mantener un concepto realista de cada uno, de manera tal que se mantenga la integridad estructural y el funcionamiento de cada uno, así como la capacidad de identificar cualquier anomalía o alteración de la estructura y funcionamiento de uno mismo. Por lo mencionado, no se realizó ninguna modificación al ítem, ya que se sustenta a la luz de la teoría. 


\section{Revista Electrónica Enfermeria Actual en costa Rica}

\section{- Validación de contenido}

La prueba de Kaiser-Meyer-Olkin (KMO) dio un índice de 0,62, dato aceptable, mientras que la prueba de esfericidad de Bartlett resultó estadísticamente significativa con un valor de $\mathrm{p}<0,000 ; 276 \mathrm{gl}$ y un Chi de 587,1 (tabla 1), lo cual favoreció el análisis para determinar los factores subyacentes de la matriz de correlación (Martínez, 2011).

El estudio factorial mediante el método ACP con rotación Varimax sugirió el análisis con 5 ejes factoriales que explicaron el $60,03 \%$ de la varianza total, cuyo criterio fue asignar un ítem al que presentara la mayor carga factorial dentro de estos ejes.

- Factor 1 (F1): Este factor arrojó el 17,12\% de la varianza total e incluyó los ítems 1, 2, 5, 9, 13, 15 y 23. Cuando hay un factor que explica más varianza significa que es el elemento que mide más dispersión, en otras palabras, él es el que mejor va a explicar las diferencias entre los distintos individuos de la muestra que se seleccionó, ya que estos factores son instrumentos que miden la dispersión. Según los resultados, dentro de este factor predominaron los ítems referentes al aspecto de la teoría de Orem (1983) relacionado con la prevención de peligros contra la vida, el funcionamiento y bienestar humano en personas que son conocidas sanas, por ende, es de suma importancia tomar medidas que cumplan con el objetivo de promover la salud y prevenir la enfermedad para evitar futuras complicaciones y que mantengan un estado de salud adecuado.

Orem (1983) menciona que dentro de los cuidados referentes a la prevención primaria, existen tres niveles, el primario, secundario y el terciario, los cuales están estrechamente relacionados con una población sana en la que el autocuidado va dirigido justamente a mantener la salud y el bienestar y la prevención de enfermedades. Dentro de este factor, también se incluye el único ítem encargado de medir el requisito universal referente al aspecto del mantenimiento de una ingesta suficiente de comida, lo cual se sabe que es necesario para el adecuado funcionamiento y desarrollo del organismo, al igual que el ítem 13 que es uno de los encargados de medir el requisito universal del mantenimiento de un equilibrio entre actividad y descanso (Orem, 1983), de lo que colige una diferencia de variables relacionadas entre sí.

- Factor 2 (F2): En este factor se dio un 14,67\% de la varianza total y contiene los ítems 4, 10, 14 16, 17 y 21 de la escala. En este factor se da una marcada presencia de ítems relacionados con la medición del requisito de prevención de peligros contra la vida, el funcionamiento y bienestar personal (5 ítems) y una única pregunta de la escala encargada de valorar la promoción del funcionamiento y desarrollo humano dentro de grupos sociales. Dentro de la teoría de Orem (1983), las definiciones de ambos requisitos universales se encuentran estrechamente relacionadas, dado que ambas buscan alcanzar la integridad humana y proveer a los individuos de herramientas que les permitan un desarrollo adecuado; la misma autora aclara que es fundamental comprender las relaciones entre los requisitos de autocuidado por parte de los profesionales en Enfermería.

- $\quad$ Factor 3 (F3): Con este factor se incorporó un 12,41\% de la varianza total y contiene los ítems 3, 7, 12, 22 y 24. Se agrupó los dos ítems encargados de medir el requisito universal del mantenimiento de un 
equilibrio entre la soledad y la interacción social, el cual es muy relevante según Orem (1983), puesto que este equilibrio proporciona a la persona condiciones que son esenciales para los procesos de desarrollo, de modo que establece una relación con los demás ítems agrupados en este factor encargados de valorar la promoción del funcionamiento y desarrollo humano dentro de grupos sociales.

- $\quad$ Factor 4 (F4): Incluyó el 8,32\% de la varianza total e incorpora los ítems 8, 18 y 19 de la escala. Al igual que el factor 2 , en este predominó la medición del requisito de prevención de peligros contra la vida, el funcionamiento y bienestar humano.

Factor 5 (F5): Representa el 7,49\% de la varianza total, abarca los ítems 6, 11 y 20. Este es el único dominio en el cual todos los ítems referentes a la valoración del mantenimiento de un equilibrio entre actividad y descanso quedaron agrupados. Orem (1983) señala que la importancia de este requisito radica en que "regula los estímulos ambientales y suministra ideas variadas, oportunidad de manifestar intereses y talentos, así como la sensación de bienestar que acompaña a ambos” (p.66). 
Tabla 1

Matriz de cargas factoriales para los ejes factoriales según análisis de componentes principales y rotación Varimax para la población conocida sana, 2011

$(\mathbf{n}=\mathbf{1 0 5})$

\begin{tabular}{|c|c|c|c|c|c|}
\hline & \multicolumn{5}{|c|}{ Factor } \\
\hline & 1 & 2 & 3 & 4 & 5 \\
\hline 15. Yo examino mi cuerpo para ver si hay algún cambio. & 811 & 244 & ,199 &,- 104 &,- 027 \\
\hline 23. Puedo sacar tiempo para mí. &, 759 &, 159 &, 167 &, 150 &, 127 \\
\hline $\begin{array}{l}\text { 1. A medida que cambian las circunstancias, yo voy haciendo } \\
\text { ajustes para mantener mi salud. }\end{array}$ &, 728 & ,221 & ,361 &, 129 & ,126 \\
\hline 13. Puedo dormir lo suficiente como para sentirme descansado. & ,699 & ,095 & ,092 &, 000 & ,265 \\
\hline $\begin{array}{l}\text { 2. Reviso si las actividades que practico para mantenerme con } \\
\text { salud son buenas. }\end{array}$ & ,698 &, 007 &, 179 & ,482 &,- 018 \\
\hline $\begin{array}{l}\text { 5. Hago, en primer lugar, lo que sea necesario para mantenerme } \\
\text { con salud. }\end{array}$ &, 591 & ,269 &, 151 & 483 &, 107 \\
\hline $\begin{array}{l}\text { 9. Para mantener el peso que me corresponde, hago cambios en mis } \\
\text { hábitos alimenticios. }\end{array}$ & ,464 & ,382 &,- 278 &,- 098 &,- 171 \\
\hline $\begin{array}{l}\text { 14. Cuando obtengo información sobre mi salud, pido } \\
\text { explicaciones sobre lo que no entiendo. }\end{array}$ &, 101 &, 813 & ,209 & 041 &, 083 \\
\hline $\begin{array}{l}\text { 17. Cuando tengo que tomar una nueva medicina, cuento con una } \\
\text { persona que me brinda información sobre los efectos secundarios. }\end{array}$ & ,099 & ,763 & 062 &,- 064 &,- 060 \\
\hline $\begin{array}{l}\text { 21. Si mi salud se ve afectada, yo puedo conseguir la información } \\
\text { necesaria sobre qué hacer. }\end{array}$ & 199 & 640 & ,175 & ,308 & 194 \\
\hline $\begin{array}{l}\text { 16. He sido capaz de cambiar hábitos -que tenía muy arraigados- } \\
\text { con tal de mejorar mi salud. }\end{array}$ & ,397 &, 508 &, 061 &, 145 & ,062 \\
\hline $\begin{array}{l}\text { 10. Cuando hay situaciones que me afectan, yo las manejo de } \\
\text { manera que pueda mantener mi forma de ser. }\end{array}$ &, 171 & ,506 &,- 140 &,- 002 &,- 123 \\
\hline $\begin{array}{l}\text { 4. Yo puedo hacer lo necesario para mantener limpio el ambiente } \\
\text { donde vivo. }\end{array}$ & ,327 &, 500 &, 122 &,- 105 & ,352 \\
\hline $\begin{array}{l}\text { 24. A pesar de mis limitaciones para movilizarme, soy capaz de } \\
\text { cuidarme como me gusta. }\end{array}$ & ,134 & ,037 &, 836 & ,121 &,- 011 \\
\hline 12. Cuando necesito ayuda, puedo recurrir a mis amigos siempre. & ,272 & ,021 & ,736 & ,049 & 059 \\
\hline 22. Si yo no puedo cuidarme, puedo buscar ayuda. &, 123 & ,237 & 693 & ,227 &, 151 \\
\hline $\begin{array}{l}\text { 3. Si tengo problemas para moverme o desplazarme, me las arreglo } \\
\text { para conseguir ayuda. }\end{array}$ & ,211 &,- 312 & 640 &,- 020 &,- 030 \\
\hline $\begin{array}{l}\text { 7. Yo puedo buscar mejores acciones para cuidar mi salud que las } \\
\text { que tengo ahora. }\end{array}$ &,- 007 & ,292 &, 564 &,- 073 &,- 033 \\
\hline 8. Cambio la frecuencia con que me baño para mantenerme limpio. &, 050 & ,245 & 013 &,- 739 &,- 158 \\
\hline $\begin{array}{l}\text { 18. Soy capaz de tomar medidas para garantizar que mi familia y } \\
\text { yo no corramos peligro. }\end{array}$ &, 229 &, 500 &, 120 &, 588 &,- 122 \\
\hline $\begin{array}{l}\text { 19. Soy capaz de evaluar qué tanto me sirve lo que hago para } \\
\text { mantenerme con salud. }\end{array}$ & ,399 & ,281 &, 173 &, 516 &, 015 \\
\hline $\begin{array}{l}\text { 20. Debido a mis ocupaciones diarias, me resulta difícil sacar } \\
\text { tiempo para cuidarme. }\end{array}$ & 269 &, 081 & 083 &, 003 &, 750 \\
\hline 6. Me faltan las fuerzas necesarias para cuidarme como debo. &,- 090 & ,221 &, 114 & ,325 & ,690 \\
\hline $\begin{array}{l}\text { 11. Pienso en hacer ejercicio y descansar un poco durante el día } \\
\text { pero no llego hacerlo. }\end{array}$ &, 076 &,- 246 &,- 099 &,- 038 & ,598 \\
\hline
\end{tabular}

Fuente: Cubillo, Porras, Ramírez y Sirias (2011). 


\section{- Consistencia interna y confiabilidad}

Los datos resultan confiables, ya que arrojaron un valor del Alfa de Cronbach de 0,84 (84\%), lo que significa que el total de los ítems de la escala logran medir un mismo concepto o se correlacionan positivamente entre sí, independientemente del tipo de contenido (Ruíz, 2011).

La tabla siguiente muestra que el ítem 8 de la escala, con respecto a la correlación elemento-total, da un resultado negativo, mas si a este se le eliminara el Alfa de Cronbach aumentaría un 0,016 y quedaría un total final de $0,860(86 \%)$ y un grado de confiabilidad aún mayor, sin embargo, no afecta su conservación ya que los valores recomendados para estos índices están entre 0.7 y 0.9 (70\% y 90\%) (Campo y Oviedo, 2008).

Tabla 2

Medición de la confiabilidad global y de cada ítem de la escala ASA, adaptada para la población costarricense, 2011

\begin{tabular}{lcccc}
\hline \hline Ítem & $\begin{array}{c}\text { Media de la } \\
\text { escala si se } \\
\text { elimina el } \\
\text { elemento }\end{array}$ & $\begin{array}{c}\text { Varianza de la } \\
\text { escala si se } \\
\text { elimina el } \\
\text { elemento }\end{array}$ & $\begin{array}{c}\text { Correlación } \\
\text { elemento- } \\
\text { total } \\
\text { corregida }\end{array}$ & $\begin{array}{c}\text { Alfa de Cronbach si se } \\
\text { elimina el elemento }\end{array}$ \\
\hline 1. & 74,73 & 74,332 &, 649 &, 830 \\
2. & 74,85 & 72,515 &, 636 &, 828 \\
3. & 74,96 & 76,402 &, 331 &, 841 \\
4. & 74,30 & 79,268 &, 410 &, 839 \\
5. & 74,70 & 76,422 &, 514 &, 835 \\
6. & 74,66 & 79,458 &, 214 &, 844 \\
7. & 74,98 & 79,327 &, 202 &, 845 \\
8. & 74,97 & 83,143 &,- 070 &, 861 \\
9. & 75,22 & 75,173 &, 433 &, 837 \\
10. & 74,78 & 78,057 &, 351 &, 840 \\
11. & 75,50 & 81,041 &, 056 &, 853 \\
12. & 74,83 & 76,451 &, 343 &, 840 \\
13. & 74,92 & 76,110 &, 388 &, 838 \\
14. & 74,47 & 75,828 &, 567 &, 833 \\
15. & 74,76 & 74,414 &, 560 &, 832 \\
16. & 74,75 & 75,150 &, 551 &, 833 \\
17. & 74,90 & 74,056 &, 467 &, 835 \\
18. & 74,38 & 78,392 &, 440 &, 838 \\
19. & 74,62 & 76,238 &, 557 &, 834 \\
20. & 75,21 & 75,590 &, 386 &, 839 \\
21. & 74,54 & 77,924 &, 482 &, 837 \\
22. & 74,63 & 76,216 &, 498 &, 835 \\
23. & 74,80 & 73,315 &, 602 &, 830 \\
24. & 74,64 & 76,368 &, 476 &, 835 \\
\hline \hline
\end{tabular}

Fuente: Cubillo, Porras, Ramírez y Sirias(2011). 


\section{DISCUSIÓN}

Para iniciar la discusión es importante destacar lo mencionado por Sánchez y Echeverry (2004), quienes proponen que un instrumento, una vez que se tiene la versión traducida, capte a un grupo de 10 a 15 personas por parte de dos o tres evaluadores distintos, cuyas partes deben contar con características similares al escenario en el que se va a aplicar finalmente la escala y se van a analizar aspectos de los rubros de la misma. Entre los rubros hay que tener en cuenta aspectos como que se logre comprender todos los componentes de la escala, que no haya ambigüedad en los términos que se van a utilizar, que se aporte variabilidad al instrumento en la medida que no más del $95 \%$ de las personas a las que se les aplique el instrumento califiquen igual un ítem, o por el contrario, si tiene varias opciones de respuesta y ninguno de los participantes utiliza alguna opción de esta respuesta, se puede eliminar esta opción de medición. En cuanto a la utilidad, se mide que el tiempo de aplicación sea adecuado, que no se necesite mucho entrenamiento para poder aplicarlo, que el instrumento sea fácil de llenar y que sea fácil de calificar el puntaje final.

Con respecto al epígrafe mencionado, y como se presentó en el apartado anterior, se modificó la estructura gramatical de varios ítems para que estos tuviesen consistencia y pertinencia, no solo en cuanto a la cultura de la población costarricense adulta, sino a que reflejara los postulados de la teoría de Autocuidado de Orem (1983).

Es importante acotar que del resultado de los dominios identificados, se puede destacar que en la mayoría de ellos predomina la categoría relacionada con el requisito universal de prevención de peligros contra la vida, el funcionamiento y bienestar humano (Orem, 1995), uno de los requisitos principales tomado en cuenta a la hora de elaborar la escala ASA.

Los resultados de la validación de apariencia, contenido y consistencia interna demuestran que esta escala es una herramienta que puede ser utilizada en cualquier nivel de atención de salud existente, ya sea como una medida complementaria para establecer un diagnóstico en términos de la capacidad de autocuidado -respecto de fortalecer la capacidad de una persona en aras de la promoción de la salud- o para tomar medidas correctivas, de acompañamiento y educación según los resultados que se muestren tras aplicar la escala a la persona que se le esté brindando los servicios profesionales en Enfermería.

En este sentido, validar la escala ASA en Costa Rica determinó la pertinencia cultural y social del lenguaje utilizado para hacer un uso confiable de esta, máxime partiendo desde el punto de vista de que no hay referentes de una validación de apariencia y contenido para esta escala, que haya sido realizada en Costa Rica, cuya beneficiaria será la población por la facilidad de elegir una mejor forma terapéutica de intervención que toma en cuenta su capacidad de autocuidado, mientras se convierten en partícipes activos del proceso.

En este proceso, el profesional en Enfermería representa un verdadero gerente de cuidado en cuanto a la instrucción y orientación que brinda no solo a la persona, sino a su familia y entorno, de modo que se brinda un cuidado holístico, sentido en el que la escala representa un instrumento que otorgue un diagnóstico de la capacidad de agencia de autocuidado de la persona a la que se le está brindado el servicio a quien, en caso de encontrarse hospitalizada, le servirá como recurso para planear la educación y acompañamiento, para mejorar el autocuidado sin que requiera la presencia permanente del profesional de Enfermería (Vargas y Fernández, 2011). 


\section{Revista Electrónica Enfermeria actualen costa Rica}

Sumado a lo anterior, la validación permite a la profesión adquirir una mayor autonomía y capacidad de trabajo multidisciplinar, interdisciplinar y transdisciplinar en la atención que se brinde a los usuarios del sistema de salud costarricense, mientras contribuye con la disciplina de enfermería en su proceso de atención en cuanto a la capacidad de hacer valer un criterio científico y pertinente, basado y sustentado por una teoría propia con la que se establezca si una persona es capaz de cuidarse a sí o no y el nivel de capacidad de agencia de autocuidado que presente.

Por las razones mencionadas, la implementación de esta escala en Costa Rica representará un avance significativo mayoritariamente desde el punto de vista cualitativo en la práctica clínica y en el desarrollo teórico de una teorizante que es propia de enfermería, los cuales posibilitarán que dichos conceptos se lleven a la práctica. (Orem, 1995).

\section{CONCLUSIONES}

- Los resultados del proceso de validación de apariencia permitieron modificar y adaptar la escala en aras de su mejor comprensión.

- La inclusión de personas adultas conocidas sanas posibilita evaluar su capacidad de agencia de autocuidado con la escala ASA, así como generar diagnósticos propios de la disciplina y determinar estrategias de intervención.

- El análisis factorial arrojó 5 ejes factoriales y afirmó que el instrumento cuenta con una adecuada validez de contenido, con propiedades psicométricas válidas y adecuadas.

- Se demostró una alta confiabilidad tras la obtención de un coeficiente Alfa de Cronbach de 0,84, lo cual refleja que los 24 miden un mismo concepto.

- En Costa Rica, la enfermería puede contar con un instrumento válido y confiable para medir la capacidad de agencia de autocuidado en las personas conocidas sanas que se encuentran en la etapa de la adultez.

\section{REFERENCIAS BIBLIOGRÁFICAS}

Achury, S. D; Sepúlveda, C; Rodríguez, S (2008) Capacidad de Agencia de Autocuidado en personas con hipertensión arterial. Revista de Enfermería. Rev. Investigación en Enfermería: Imagen y Desarrollo 10 (1): 93-111

Álvarez, R. (1994). Estadística multivariante y no paramétrica con SPSS. Madrid: Díaz de Santos. (Versión en línea) Consultado el 25 de Marzo, 2013. Disponible en http://books.google.co.cr/books?id=GxhpROTHB0C\&pg=PA237\&dq=an $\% \mathrm{C} 3 \%$ A 1 lisis + factorial + rotaci $\% C 3 \% B 3 n+$ Varimax $\& 1 \mathrm{r}=\# \mathrm{PPP} 1, \mathrm{M} 1$

Campo, A; Oviedo, H. (2008). Propiedades psicométricas de una escala: la consistencia interna. Revista Salud Pública, Vol. 10, $\mathrm{N}^{\circ}$. (Versión en línea) Consultado el 10 de enero del 2014. Disponible en http://www.scielosp.org/scielo.php?pid=S012400642008000500015\&script=sci_abstract

Contreras, A; Flórez, I; Herrera, A. (2008). Un instrumento para evaluar la adherencia: su validez facial y confiabilidad. Cartagena, Colombia. (Versión en línea). Consultado el 15 de Noviembre del 2013. Disponible en http://www.enfermeria.unal.edu.co/revista/articulos/xxvi2_4.pdf 
Espitia, C. (2011). Asociación de los factores básicos condicionantes del autocuidado y la capacidad de agencia de autocuidado en personas con hipertensión arterial que asisten a consulta externa del hospital universitario la samaritana. Tesis para optar el título de Magíster en Enfermería con énfasis en énfasis en cuidado para la salud cardiovascular.

Evers, G. (1998). Meten van zelfzorg. Verpleegkundige instrumenten. Voor onderzoek en klinische praktijk. Leuven University Press.

Fernández, A. y Manrique, F. (2010). Efecto de la intervención educativa en la agencia de autocuidado del adulto mayor hipertenso de Boyacá, Colombia, Suramérica. Ciencia y Enfermería XVI (2). (Versión en línea). Consultado el 10 de enero del 2014. Disponible en: http://www.scielo.cl/pdf/cienf/v16n2/art_09.pdf

Gallegos, E. (1998).Validez y confiabilidad de la versión en español de la escala: Valoración de las Capacidades de Autocuidado. Desarrollo científico de Enfermería; 6(9): 260-266

George, D; Mallery, P. (1995). SPSS/PC + Step by: A simple guide and reference. Belmont, CA: Wadsworth Publishing Company.

Hernández, R; Fernández C; Baptista, P. (2006). Metodología de la Investigación. 4ta ed. México, D.F.: Ed. McGraw Hill Interamericana.

Kerlinger, F; Lee, H. (2002). Investigación del comportamiento. Métodos de investigación en Ciencias Sociales. México D.F.: McGraw-Hill,

Lamprea, J; Gómez, C. (2007). Validez en la Evaluación de Escalas. Revista Colombiana de Psiquiatría. Asociación Colombiana de Psiquiatría. Bogotá, Colombia. (Versión en línea) Consultado el 10 de enero de 2012. Disponible en: http://redalyc.uamex.mx

Leiva, V. (2009). ¿Por qué enseñar bioética social y de salud pública en Enfermería? Revista Enfermería Actual en Costa Rica. $\mathrm{N}^{\mathrm{o}}$ 17. (Versión en línea) Consultado el 10 de enero del 2014. Disponible en http://redalyc.uaemex.mx/redalyc/html/448/44812828001/44812828001_1.html

Manrique, F., Fernández, A., Velandia, A. (2009). Análisis factorial de la escala Valoración de Agencia de Autocuidado (ASA) en Colombia. Revista Aquichan vol9, n3.

Martínez, E. (2011). Análisis Factorial. 2da Canarias, España.: Ed. Editorial Contreras.

Monge, Ch. (2004). Comparación de la capacidad de agencia de autocuidado en dos grupos de mujeres adultas: uno de ellos con hipertensión arterial y el otro con enfermedad cardiovascular en el Hospital Mario Catarino Rivas, San Pedro Sula. Honduras. Universidad Nacional de Colombia. Bogotá, Colombia.

Morales, P. (2011). El Análisis Factorial en la construcción e interpretación de test, escalas y cuestionarios. Universidad Pontífica Comillas. Madrid, España.

Orem, D. (1983). Normas prácticas en Enfermería. Madrid, España: Ediciones Pirámide.

Orem, D. (1995). Nursing: Concepts of practice. Fifth edition. Mosby Missouri, United States. 
Organización Panamericana de la Salud/Organización Mundial de la Salud. (2007). Renovación de la Atención Primaria de Salud en las Américas. Documento de posición de la Organización Panamericana de la Salud/ Organización Mundial de la Salud.

Ortiz, G. (2002) Factores básicos condicionantes del autocuidado asociados a la capacidad potencial de autocuidarse para promoción de la salud de personas de 18 años y más, que viven en situación de enfermedad cardiovascular y que asisten a consulta al centro de salud Pedro Altamirano, Nicaragua. Universidad Nacional de Colombia. Bogotá, Colombia.

Papalia, D. (2005). Desarrollo Humano. Novena Edición. Mc-Graw Hill. México.

Porras, M. (2002) Factores básicos condicionantes del autocuidado asociados a la capacidad potencial de autocuidarse y promover la salud de personas de 18 años y más, que viven en situación de enfermedad cardiovascular y asisten a la consulta externa del Hospital Roosevelt en la ciudad de Guatemala. Universidad Nacional de Colombia. Bogotá, Colombia.

Real Academia Española (2011) http://www.rae.es/rae.html Consultado el 22 de noviembre del 2012.

Rivera, A; Díaz, L. (2007). Relación entre la capacidad de agencia de autocuidado y los factores de riesgo cardiovascular. Colombia. Revista Cuadernos Hospital de Clínicas, Vol. 52, n2. (Versión en línea) Consultado el $1^{\circ}$ de julio del 2013.2 Disponible en: http://www.docentes.unal.edu.co/lnriveraa/docs/RELACION\%20ENTRE\%20LA\%20CAPACIDAD\%20DE\%20A GENCIA\%20DE\%20AUTOCUIDADO\%20Y\%20LOS\%20FACTORES\%20DE\%20RIESGO\%20CARDIOVASC ULAR.pdf

Rivera, A. (2006) Capacidad de Autocuidado en personas con hipertensión arterial hospitalizadas en una clínica de Bogotá, Colombia. Revista de Salud Publica, Vol. 8 n 3.

Rodríguez, M. y Díaz, R. (2011) Instrumento para medir las capacidades de autocuidado en los pacientes adultos hospitalizados con terapia intravenosa. (Versión en línea) Diversidad Cultura y Salud. Consultado el 10 de enero de 2012.

Disponible

en:

http://www.eumed.net/libros/2011f/1142/instrumento_para_medir_las_capacidades_de_autocuidado_en_los_pacien tes_adultos_hospitalizados_con_terapia_intravenosa.html

Ruiz, C. (2011). Confiabilidad. Universidad Pedagógica Experimental Libertador. Maracaibo, Venezuela. Recuperado de: http://investigacion.upeu.edu.pe/images/7/74/Validez.pdf

Sánchez, R; Echeverri, J. (2004). Validación de escalas en medición de salud. Revista de Salud Pública, Colombia.

Vargas B., Fernández, C. (2011) Programa de alta hospitalaria programada para preparar el egreso del paciente y familia. Rev. Enfermería Actual en Costa Rica [en línea].21, 1-20 [citado (noviembre 2013)]. Disponible en $<$ http://www.revenf.ucr.ac.cr/programa.pdf /ISSN 1409-4568

Velandia, A., Rivera, A. (2009) Confiabilidad de la escala de "Apreciación de la agencia de autocuidado (ASA) segunda versión en español adaptada para población colombiana”. Revista avances en enfermería, vol. 27 n1. 